\title{
A STRUCTURE THEOREM FOR STATIONARY PERFECT FLUIDS
}

\author{
BRENDAN GUILFOYLE
}

\begin{abstract}
It is proven that, under mild physical assumptions, an isolated stationary relativistic perfect fluid consists of a finite number of cells fibred by invariant annuli or invariant tori. For axially symmetric circular flows it is shown that the fluid consists of cells fibred by rigidly rotating annuli or tori.
\end{abstract}

\section{INTRODUCTION}

The purpose of this paper is to extend some results in the non-relativistic theory of Euler flows to the theory of stationary perfect fluids in general relativity. The latter play an important role in understanding the Einstein field equations in the presence of matter, while the former sits within the larger field of topological hydrodynamics. In particular, our main result is:

Theorem 1: Consider an analytic stationary perfect fluid flow on an analytic spacetime $\left({ }^{4} M,{ }^{4} g\right)$ with proper pressure $p$ and density $\mu$ satisfying Einstein's field equations, which has the following attributes:

(a) it is spatially compact,

(b) it satisfies the weak energy condition: $p+\mu>0$,

(c) it is isentropic: $\mu=\mu(p)$,

(d) it is non-Beltrami (see Definition 3 below).

Then the spatial fluid region is divided into a finite number of cells of two types: if the cell does not intersect the boundary it is fibred by tori invariant under the flow, if the cell intersects the boundary it is fibred by annuli invariant under the flow. The flow lines on a torus are either all closed or all dense, while the flow lines on an annulus are all closed.

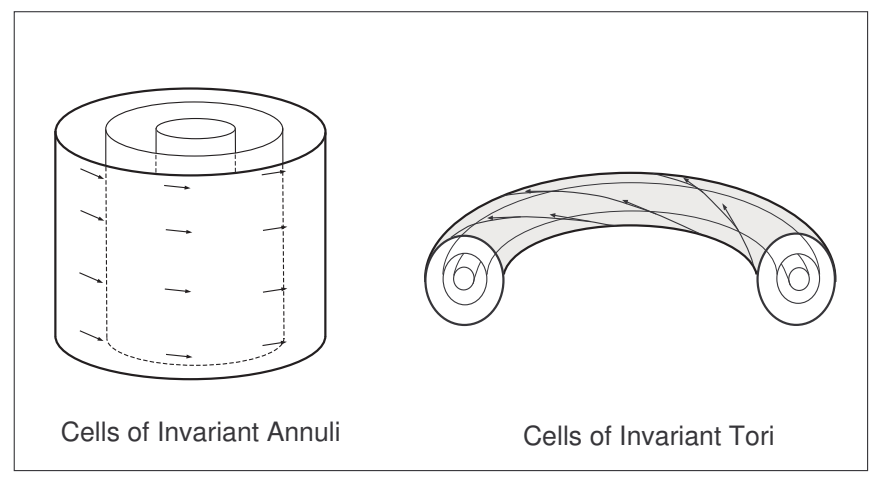

Date: February 21st, 2005.

Key words and phrases. relativistic perfect fluid, stationary. 
To prove this theorem we find a pair of commuting vector fields that generate the invariant surfaces. These invariant surfaces have less geometric significance than the surfaces of transitivity of axial symmetry, but the theorem states that they are (generically) topologically restricted. No more than the conservation equations of general relativity are needed to prove this.

We apply this theorem in the case where the fluid is axially symmetric and the fluid 4-velocity lies in the plane spanned by two Killing vectors, so called circular flows:

Theorem 2: A circular fluid flow is made up of cells foliated by rigidly rotating annuli or tori.

In the next section we summarise the background material of stationary perfect fluids needed to prove the Structure Theorem. This includes a reformulation of the conservation equations for a relativistic stationary perfect fluid along the lines of the Euler equations for a steady incompressible fluid flow. Section 3 provides the definition of relativistic Beltrami flows and the proof of Theorem 1 while section 4 applies the theorem to circular fluid flows. The final section contains a discussion of the results and their implications.

\section{Stationary Perfect Fluid Flows}

A lorentz 4 -manifold $\left({ }^{4} M,{ }^{4} g\right)$ is said to be stationary if it admits a timelike Killing vector $\xi^{\nu}$. We can choose coordinates $\left(x^{0}=t, x^{i}\right)$ on a stationary spacetime so that $\xi^{\mu}=\delta_{0}^{\mu}$ and the metric takes the $t$-independent form

$$
d s^{2}=g_{i j} d x^{i} d x^{j}-e^{\omega}\left(d t+A_{i} d x^{i}\right)^{2} .
$$

Here and throughout Latin indices run through 1,2,3, while Greek indices run through $0,1,2,3$.

Locally we can quotient out by the action of the Killing vector on ${ }^{4} M$ to get a riemannian 3-manifold $(M, g)$, which we think of as the spatial slice of the spacetime. We use this riemannian metric to raise and lower all spatial tensor indices. However, for tensors defined on the whole spacetime we will continue to use ${ }^{4} g$. For example, since $U^{\mu}$ is a 4 -vector

$$
U_{i}={ }^{4} g_{i \nu} U^{\nu}={ }^{4} g_{i j} U^{j}+{ }^{4} g_{i 0} U^{0}=g_{i j} U^{j}-e^{\omega} A_{i} A_{j} U^{j}+{ }^{4} g_{i 0} U^{0},
$$

while for a 3-vector $V^{i}, V_{i}=g_{i j} V^{j}$.

For later use, we note that for any $t$-independent 4-vector $U^{\mu}$ the divergence with respect to the lorentz and riemannian metrics are related by the simple formula

$$
{ }^{4} \nabla_{\mu} U^{\mu}=\nabla_{i} U^{i}+\frac{1}{2} \omega_{, i} U^{i} .
$$

For any 4-vector $U^{\mu}$ we note the following useful relations

$$
\begin{array}{cc}
U^{0}=-e^{-\omega} U_{0}-A_{i} U^{i} & U_{0}=-e^{\omega}\left(U^{0}+A_{i} U^{i}\right) \\
U^{i}=g^{i j} U_{j}-A^{i} U_{0} & U_{i}=g_{i j} U^{j}+A_{i} U_{0},
\end{array}
$$

and if $U_{\mu} U^{\mu}=-1$ we have that

$$
e^{-\omega} U_{0}^{2}=1+g_{i j} U^{i} U^{j} .
$$


Consider a stationary spacetime $\left({ }^{4} M,{ }^{4} g\right)$ on which we have a perfect fluid with 4 -velocity $U^{\nu}$, pressure $p$, density $\mu$ and energy momentum tensor

$$
T_{\alpha \beta}=(p+\mu) U_{\alpha} U_{\beta}+p^{4} g_{\alpha \beta},
$$

and $U_{\nu} U^{\nu}=-1$.

The assumption that the flow is steady on a stationary spacetime means that $U^{\nu}, p$ and $\mu$ are all independent of $t$.

For such a fluid, the conservation equations ${ }^{4} \nabla^{\mu} T_{\nu \mu}=0$ read

$$
{ }^{4} \nabla_{\nu} p+2(p+\mu) U^{\alpha}{ }^{4} \nabla_{[\alpha} U_{\nu]}+\left(U^{\alpha}{ }^{4} \nabla_{\alpha}(p+\mu)+(p+\mu){ }^{4} \nabla_{\alpha} U^{\alpha}\right) U_{\nu}=0 .
$$

Multiplying this across by $U^{\nu}$ we see that

$$
(p+\mu){ }^{4} \nabla_{\alpha} U^{\alpha}+U^{\alpha}{ }^{4} \nabla_{\alpha} \mu=0 .
$$

Substituting this back into (2.1) we get that

$$
{ }^{4} \nabla_{\nu} p+2(p+\mu) U^{\alpha}{ }^{4} \nabla_{[\alpha} U_{\nu]}+U_{\nu} U^{\alpha}{ }^{4} \nabla_{\alpha} p=0 .
$$

Now taking the $\nu=0$ component we get

$$
U_{0} U^{i} \nabla_{i} p+(p+\mu) U^{i} \nabla_{i} U_{0}=0,
$$

while taking the $\nu=i$ component we get

$$
\nabla_{i} p+2(p+\mu) U^{j} \nabla_{[j} U_{i]}-(p+\mu) U^{0} \nabla_{i} U_{0}+U_{i} U^{j} \nabla_{j} p=0 .
$$

Multiplying this across by $U_{0}$, using $(2.3)$ and the fact that $U_{0} U^{0}=-1-U_{i} U^{i}$ we find that

$$
U_{0} \nabla_{i} p+(p+\mu) \nabla_{i} U_{0}+2(p+\mu) U_{0}^{2} U^{j} \nabla_{[j}\left(U_{0}^{-1} U_{i]}\right)=0 .
$$

Equations (2.2) and (2.4) are the relativistic Euler equations governing a steady fluid perfect fluid flow on a stationary spacetime.

Definition 1. A perfect fluid is isentropic if it has an equation of state $\mu=\mu(p)$.

In what follows we will assume that the perfect fluid is isentropic. If we introduce the real-valued functions $\alpha$ and $\beta$ on $M$ given by

$$
\ln (\alpha)=\int \frac{d p}{p+\mu} \quad \ln (\beta)=\int \frac{d \mu}{p+\mu},
$$

then the conservation equations can be written

$$
\begin{gathered}
\nabla_{i} U^{i}+\frac{1}{2} U^{i} \nabla_{i} \omega+U^{i} \nabla_{i} \ln (\beta)=0 \\
\nabla_{i} \ln \left[\alpha U_{0}\right]+2 U_{0} U^{j} \nabla_{[j}\left(U_{0}^{-1} U_{i]}\right)=0 .
\end{gathered}
$$

Definition 2. An isolated stationary body is one for which the spatial set $V \subset M$ on which the energy momentum tensor is non-zero is compact, and the Darmois conditions hold on the boundary [1].

For a perfect fluid (with $p+\mu \neq 0$ ) being isolated implies the vanishing of the pressure and tangency of the fluid flow at the boundary. 


\section{The Structure Theorem}

Consider the two vector fields

$$
X^{i}=U_{0} U^{i} \quad Y^{i}=U_{0} \beta^{-1} e^{-\frac{1}{2} \omega} \eta^{i j k} \nabla_{[j}\left(U_{0}^{-1} U_{k]}\right),
$$

where $\eta$ is the hodge star operator:

$$
\eta^{i j k}=\frac{1}{\sqrt{\operatorname{det} g}} \epsilon^{i j k}
$$

$\epsilon^{i j k}$ being the 3-dimensional Levi-Civita permutation symbol.

Definition 3. We say that the flow is Beltrami if $Y^{i}=\lambda X^{i}$ for some real-valued function $\lambda$ on $V$.

The reason for such a definition becomes clear from the following structure theorem for relativistic isentropic flows:

Theorem 1. Consider a stationary analytic perfect fluid flow on an analytic spacetime that:

(a) is isolated,

(b) satisfies the weak energy condition $p+\mu>0$,

(c) is isentropic: $\mu=\mu(p)$,

(d) is non-Beltrami.

Then the spatial fluid region is divided into a finite number of cells of two types: if the cell does not intersect the boundary it is fibred by tori invariant under the flow, if the cell intersects the boundary it is fibred by annuli invariant under the flow. The flow lines on a torus are either all closed or all dense, while the flow lines on an annulus are all closed.

Proof. The key to the proof is to show that the vector fields $X^{i}$ and $Y^{i}$ commute. To show this we use the 3 -dimensional vector notation

$$
\begin{array}{cc}
\vec{U} \times \vec{V}=\eta^{i j k} U_{[j} V_{k]} \frac{\partial}{\partial x^{i}} & \operatorname{curl}(\vec{U})=\eta^{i j k} \nabla_{[j} U_{k]} \frac{\partial}{\partial x^{i}} \\
\operatorname{div}(\vec{U})=\nabla_{i} U^{i} & \operatorname{grad}(p)=g^{i j} \frac{\partial p}{\partial x^{i}} \frac{\partial}{\partial x^{j}} .
\end{array}
$$

The Euler equations (2.5) and (2.6) say that

$$
\begin{gathered}
\operatorname{div}(\vec{X})=\vec{X} \cdot \operatorname{grad}\left[-\frac{1}{2} \omega+\ln \left(U_{0}\right)-\ln (\beta)\right] \\
\operatorname{grad}\left(\ln \left[\alpha U_{0}\right]\right)-\vec{X} \times \operatorname{curl}(\vec{T})=0,
\end{gathered}
$$

where we have introduced the 3 -vectors $W^{i}=U^{i}$ and $T^{i}=g^{i j} U_{0}^{-1} U_{j}=U_{0}^{-1} W^{i}+$ $A^{i}$. To compute the commutator of $X^{i}$ and $Y^{i}$ we utilise the vector identity

$$
[\vec{X}, \vec{Y}]=\operatorname{curl}(\vec{Y} \times \vec{X})+\vec{X} \operatorname{div}(\vec{Y})-\vec{Y} \operatorname{div}(\vec{X}) .
$$

With the help of the Euler equations and the vector identities curl $(\operatorname{grad} f)=0$ and $\operatorname{div}(\operatorname{curl}(\vec{T}))=0$, this says that

$$
\begin{aligned}
{[\vec{X}, \operatorname{curl}(\vec{T})] } & =\operatorname{curl}(\operatorname{curl}(\vec{T}) \times \vec{X})+\vec{X} \operatorname{div}(\operatorname{curl}(\vec{T}))-\operatorname{curl}(\vec{T}) \operatorname{div}(\vec{X}) \\
& =-\operatorname{curl}(\vec{T}) \vec{X} \cdot \operatorname{grad}\left[-\frac{1}{2} \omega+\ln \left(U_{0}\right)-\ln (\beta)\right] .
\end{aligned}
$$


This can be rearranged to

$$
[\vec{X}, \vec{Y}]=\left[\vec{X}, U_{0} \beta^{-1} e^{-\frac{1}{2} \omega} \operatorname{curl}(\vec{T})\right]=0,
$$

as required.

The rest of the proof now follows that of the non-relativistic case and therefore we only sketch it below - details can be found in [2].

By analyticity, the critical set $K$ of the function $\ln \left(\alpha U_{0}\right)$ forms an semianalytic subset and $M \backslash K$ has a finite number of cells.

Assume that $X^{i}$ and $Y^{i}$ are not everywhere collinear. We can conclude that at non-critical values for $\ln \left(\alpha U_{0}\right)$, the level sets form an oriented smooth 2-manifold with an $\mathbb{R}^{2}$ action. If the level set does not intersect $\partial V$, it forms a torus with all flow lines closed or all flow lines dense. If it does intersect the boundary, it must form an annulus with closed flowlines.

Note: A similar Structure Theorem holds in the case of dust, where the pressure is identically zero.

\section{Axially Symmetric Flows}

When one considers the definition of axial symmetry for a stationary field, there are a number of reasonable possibilities [3] [4] [5]. For our purposes we use the following definition:

Definition 4. A stationary space-time $\left({ }^{4} M,{ }^{4} g\right)$, with timelike Killing vector $\xi^{\nu}$, is axially symmetric if there exists a spacelike Killing vector $\eta^{\nu}$ that has closed orbits and commutes with $\xi^{\nu}$.

Now suppose we have a stationary, axisymmetric spacetime $\left({ }^{4} M,{ }^{4} g\right)$ as defined above. The simplest type of flow appears to be one where the spatial fluid flow $U^{i}$ is parallel to $\eta^{i}$.

Definition 5. A fluid flow on a stationary axisymmetric spacetime is circular if the fluid 4 -velocity $U^{\nu}$ lies in the plane spanned by the Killing vectors $\xi^{\nu}$ and $\eta^{\nu}$. In addition we assume that the spacetime has a regular axis of symmetry [6] [7].

Proposition 1. A circular flow is Beltrami iff $\lambda=0$.

Proof. Working locally there exist coordinates $\left(x^{a}, \phi, t\right)$ such that the Killing vectors are

$$
\vec{\xi}=\frac{\partial}{\partial t} \quad \vec{\eta}=\frac{\partial}{\partial \phi}
$$

We now utilise a remarkable theorem [6] [7] that says that for a circular fluid flow, the 2-planes orthogonal to the Killing orbits generated by $\xi^{\nu}$ and $\eta^{\nu}$ are integrable. Thus the metric can be reduce to

$$
d s^{2}=h_{a b} d x^{a} d x^{b}+H^{2} d \phi^{2}-e^{\omega}(d t+A d \phi)^{2},
$$

where all of the components of the metric are independent of both $t$ and $\phi$.

Circularity means that the fluid flow vector is given by

$$
U^{\nu}=U^{0}\left(\xi^{\nu}+\Omega \eta^{\nu}\right),
$$

for some function $\Omega$. In particular, the only non-vanishing component of the spatial vector $\vec{X}$ is

$$
X^{\phi}=U_{0} U^{0} \Omega .
$$


On the other hand

$$
Y^{\phi}=U_{0} \beta^{-1} e^{-\frac{1}{2} \omega}(\operatorname{det}(g))^{-\frac{1}{2}} \epsilon^{b c \phi} \partial_{[b}\left(U_{0}^{-1} W_{c]}+A_{c]}\right)=0 .
$$

This means that $\vec{X}$ and $\vec{Y}$ are orthogonal, and so the flow is Beltrami iff $\vec{Y}=0$.

Virtually all results on stationary axially symmetric perfect fluids, whether exact solutions, asymptotic behaviour or a priori estimates, pertain only to circular flows. Thus the metric has the form (4.1) and the fluid velocity (4.2).

Definition 6. The function $\Omega$ defined in equation (4.2) is called the angular velocity of the fluid. A spinning fluid body with constant angular velocity is said to be in rigid rotation. If the angular velocity is not constant, the fluid is said to have differential rotation.

Theorem 2. A circular flow consists of cells foliated by rigidly rotating annuli or tori.

Proof. First, we gather together some of the relationships enjoyed by the angular velocity, the spatial fluid velocity vector $W^{i}$, the 4-components of the fluid $U^{0}$ and $U_{0}$, and the metric functions $H, e^{\omega}$ and $A$.

$$
\begin{gathered}
U_{0}=-(1+A \Omega) e^{\omega} U^{0}=\sqrt{1+|W|^{2}} e^{\frac{1}{2} \omega} \\
W^{\phi}=U^{0} \Omega \quad W_{\phi}=\frac{|W|^{2}}{U^{0} \Omega} \\
H^{2}=\frac{|W|^{2}}{\left(U^{0} \Omega\right)^{2}} \quad U^{0} U_{0}=-\frac{1+|W|^{2}}{1+A \Omega} \\
A=-\left(\frac{1}{\Omega}+\frac{1+|W|^{2}}{U^{0} U_{0} \Omega}\right),
\end{gathered}
$$

where we have introduced $|W|^{2}=g_{i j} W^{i} W^{j}$.

Now, introduce coordinates $(\rho, z)$ on the 2 -space orthogonal to the group orbits, so that the commuting vector fields are

$$
\vec{X}=X^{\phi} \frac{\partial}{\partial \phi} \quad \vec{Y}=Y^{z} \frac{\partial}{\partial z} .
$$

Here $\rho$ labels the invariant surfaces and $(z, \phi)$ are coordinates on the invariant surfaces, analogous to the action angles of classical mechanics. In these coordinates the Euler equations dramatically simplify to

$$
\begin{gathered}
\frac{\partial}{\partial z}\left(U^{0} U_{0} \Omega\right)=0 \quad \frac{\partial}{\partial z}\left(A+\frac{|W|^{2}}{U^{0} U_{0} \Omega}\right)=0 \\
\frac{\partial}{\partial z}\left(\alpha U_{0}\right)=0 \quad \frac{\partial}{\partial \rho}\left(\alpha U_{0}\right)=U^{0} U_{0} \Omega \frac{\partial}{\partial \rho}\left(A+\frac{|W|^{2}}{U^{0} U_{0} \Omega}\right) .
\end{gathered}
$$

The first two of these, along with the identity (4.3), tell us that the angular velocity $\Omega$ is constant on each invariant surface. Thus each annulus or torus rotates rigidly, although there may be differential rotation between the surfaces. In addition, $U^{0} U_{0}$ is constant on each invariant surface. 


\section{Discussion}

In topological (non-relativistic) hydrodynamics there is an equivalent Structure Theorem for steady incompressible perfect fluid flows on a riemannian 3-manifold [2]. The dynamics of the fluid is described by four equations: the three Euler equations and the incompressibility condition. In this setting a fluid is Beltrami if the velocity vector is everywhere collinear with it's curl. In contrast to the regular behaviour of non-Beltrami fluids guaranteed by the Structure Theorem, the flowlines of Beltrami fluids can be arbitrarily knotted [8] [9] [10] [11]. Generically a fluid will be non-Beltrami, but Beltrami fluids do arise, for example, as "force-free" fields in magneto-hydrodynamics [12].

In the relativistic setting, stationary perfect fluids have long been studied and while much progress has been made, a convincing overview has yet to emerge. The literature in this area is rather extensive and we mention just some topics pertinent to our result - for a review see [13]. In particular, there has been much progress in finding and investigating local solutions that satisfy various simplifying assumptions. These can range from the weak (like energy conditions) to the strong (like cylindrical symmetry), or a combination of both.

Without doubt the most commonly imposed conditions are those of axial symmetry and circular flow, usually supplemented by some other restriction [14] [15] [16] [17] [18]. This setting comes closest to Newtonian theory and thus we are entitled to attempt to physically interpret the geometric quantities that arise. As has been shown, however, even in this case, care must be taken [19]. A further down side is that it is not clear which of the properties these local solutions possess are just products of their specialty. Thus, failure to match globally to the vacuum Kerr solution, a difficulty that every known stationary perfect fluid possesses, may simply be an artifact of their axial symmetry or circularity.

Aside from exact solutions, there has been progress on general matching conditions with the Kerr solution [20] [21], as well as results on a priori estimates on the equatorial radius [22], positivity of angular momentum density [23] and asymptotics [24]. In all cases axial symmetry is assumed, or the fluid is assumed to have zero pressure, i.e. the body consists of dust.

It is within this context that our Structure Theorem above should be viewed. For here, the assumptions made on the fluid are nothing more than one would impose on any physically reasonable model for an isolated stationary fluid body. Moreover, such a stationary perfect fluid is precisely what is required to model the equilibrium states of rotating fluid bodies [25] [26].

Examples of both types of cells in the Theorem have appeared in the literature. The remarkable perfect fluid source for the NUT metric discovered by Lukács [27] has flowlines given by the Hopf fibering of $S^{3}$, which lie on invariant tori. The second type of cells arise, for example, when one considers cylindrically symmetric flows [28] [29] [30] [31]. The decomposition of the full Einstein field equations parallel and transverse to the foliation is worthy of careful consideration.

It is hoped that the Structure Theorem can yield a new approach to finding a source for the Kerr metric. It indicates that the simplest such source must be a solid torus and the fact that the singularity in the maximally extended Kerr solution is ring shaped supports such a conclusion.

Another point worthy of further consideration is the Beltrami condition. In the non-relativistic case, Beltrami flows exhibit chaotic dynamics and are closely 
related to contact geometry. The natural definition in the relativistic case afforded us by the Structure Theorem is a good deal more complex and its relationship with contact structures is unclear. It nevertheless raises the question as to whether relativistic effects "tame" Beltrami flows, or whether chaotic regimes persist.

\section{REFERENCES}

[1] M. Mars and J.M.M. Senovilla, Classical Quantum Gravity 10 (1993), 1865.

[2] V.I. Arnold and B.A. Khesin, Topological Methods in Hydrodynamics, Springer, New York (1998).

[3] A. Barnes, Classical Quantum Gravity 16 (1999), 919.

[4] A. Barnes, Classical Quantum Gravity 18 (2001), 5511.

[5] M. Mars and J.M.M. Senovilla, Classical Quantum Gravity 10 (1993), 1633.

[6] B. Carter, J. Math. Phys. 10 (1969), 70.

[7] W. Kundt and M. Trümper, Z. Phys. 192 (1966), 419.

[8] T. Dombre et al, J. Fluid Mech. 167 (1986), 353.

[9] J. Etnyre and R. Ghrist, Nonlinearity 13 (2000), 441.

[10] J. Etnyre and R. Ghrist, Ergodic Theory Dynam. Systems 22 (2002), 819.

[11] J. Etnyre and R. Ghrist, Trans. Amer. Math. Soc. 352 (2002), 5781.

[12] S. Chandrasekhar, Proc. Natl. Acad. Sci. USA 42 (1956), 1.

[13] F.J. Chinea and L.M. González-Romero (Eds.): Rotating Objects and Relativistic Physics, Proceedings, El Escorial, Lecture Notes in Physics Vol 423, Springer, 1993.

[14] D. Kramer et al, Exact Solutions of Einstein's Field Equations, Cambridge University Press, Cambridge, England (1980).

[15] A. Krasiński, Acta Phys. Polon. B 5 (1974), 411.

[16] A. Krasiński, Acta Phys. Polon. B 6 (1975), 223.

[17] H.D. Wahlquist, Phys. Rev. 172 (1968), 1291.

[18] F.J. Chinea and L.M. González-Romero, Classical Quantum Gravity 9 (1992), 1271.

[19] F.J. Chinea and M.J. Pareja, Classical Quantum Gravity 16 (1999), 3823.

[20] W. Roos, Gen. Relativity Gravitation 7 (1976), 431.

[21] W. Roos, Gen. Relativity Gravitation 9 (1977), 753.

[22] J. Klenk, Classical Quantum Gravity 15 (1998), 3203.

[23] R.O. Hansen and J. Winicour, J. Math. Phys. 16 (1975), 804.

[24] A. Caporali, Phys. Lett. A 66 (1978), 5.

[25] J. Ehlers, in Relativity, Astrophysics and Cosmology, W. Israel (ed.), D. Reidel, DordrechtBoston, (1973).

[26] L. Lindblom, Astrophys. J. 208 (1976), 873.

[27] B. Lukács et al, Gen. Relativity Gravitation 15 (1983), 567.

[28] W. Davidson, Classical Quantum Gravity 17 (2000), 2499.

[29] D. Sklavenites, Classical Quantum Gravity 16 (1999), 2753.

[30] J. Winicour, J. Math. Phys. 16 (1975), 1806.

[31] C.V. Vishveshwara and J. Winicour, J. Math. Phys. 18 (1977), 1280.

Brendan Guilfoyle, Department of Mathematics and Computing, Institute of Technology, Tralee, Clash, Tralee, Co. Kerry, Ireland.

E-mail address: brendan.guilfoyle@ittralee.ie 